To the authors' knowledge (information given by Cheltenham Drug Squad) this preparation after nine months has not found its way on to the black market outside of the Cheltenham area. Only one addict has admitted trying to inject the mixture: she concluded that it was "impossible," and she has not tried doing this again.

Thus it would appear that this new dipipanone mixture has considerable advantages over any other opiate preparation currently being prescribed to maintain drug addicts.

\section{JEFFREY MARKS}

J LEAvER

Cheltenham General Hospital,
Cheltenham, Glos GL53 7AN

SIR,-Dr John Strang's article (27 March, p $972)$ is interesting, strange, and at times illogical. He omits certain topics which are vital to any discussion on this subject. These include the recent increase in drug addiction and in smuggled heroin and the unknown effects of these on the black market in pharmaceutical drugs; the inability of many addicts to find a clinic to take them on, and the refusal of a large number of addicts to attend clinics even when they could; the disillusion felt by many NHS practitioners with clinic policies and practice, and the decision of some to treat their own addicts themselves; and the recent formation of an association for independent doctors who are interested in drug addiction.

Some of Dr Strang's comments on private medicine are extreme. For instance, his "prescribing doctor who follows the commercial model of providing the exact service demanded by the customer" exists only in this field I believe in an occasional, usually senile doctor who is battened on by addicts (and is usually quietly eased from the scene by the machinery that exists to deal with such a situation in drug addiction). Independent doctors who do this work (both privately and on the NHS) have banded together to form the Association of Independent Doctors in Addiction (AIDA). This association is dedicated to achieving and maintaining high standards in the field of medical practice. Independent doctors can provide much which clinics often cannot provide, including a continuous relationship with one doctor who is fully responsible for his patient. Clinics can also provide what independent doctors usually cannot provide-for example, group decisions in patient treatment, and group psychotherapy. Moreover, both have different problems to face. The private independent doctor has to face the difficulties associated with being paid by fee from patients rather than by salary and the independent NHS doctor has to cope with the problem of devoting much time to a patient without receiving any payment above the capitation fee. The clinic has other problems which cannot be enumerated here.

Clearly both clinics and independent doctors have important parts to play in coping with the present epidemic of heroin addiction. Each is often needed at different times by the same patient. This makes one of Dr Strang's suggestions extraordinary indeed. He suggests that a doctor who "chooses to take over the clinical care of a drug addict" should continue to treat him even if he clearly cannot do sofor example, if the addict disrupts his practice. This would introduce an important new principle into medical practice, not followed I think in any other branch of medicine. If every doctor who started to treat a patient was obliged to continue with that treatment regardless of what happened we would have a strange situation. Such a policy would be disastrous in medicine, surgery, or any other branch, and I believe it would be in drug addiction. It is vital that clinic and independent doctor co-operate with each other. Failure to do so along the lines suggested by Dr Strang can only harm the patients.

London W1N 1 PB

ANN DaLly

\section{Increase in drug resistance among Shigella dysenteriae, Sh flexneri, and Sh boydii}

SIR,-In a letter from Dr A S Nanivadekar and S D Gadgil (31 October, p 1187) it was proposed that the present laboratory methods of assessing and reporting bacterial resistance to antibiotics be modified since they seem to yield a high percentage of false resistant results and thus steer antibiotic usage from economical old drugs to expensive new drugs. A stuciy we did in 1980 supports this view.

We hospitalised adult patients with either lower respiratory infection (LRI-temperature $39.5 \mathrm{C}$, dyspnoea, purulent sputum, blood neutrophils $.7 \times 10^{6} / 1$, and positive chest $x$ ray signs) or urinary tract infection (UTItemperature $39.5 \mathrm{C}$, urine frequency or dysuria, albuminuria, pyuria, bacteriuria, and colony count $\left.10^{8} / 1\right)$ without anatomical defects. A physician (NBJ) personally collected samples of expectorated sputum or urine and carried them to the laboratory within an hour Culture and antibiotic sensitivity by BauerKirby method ${ }^{1}$ were done as prescribed, separately from routine hospital work, by a microbiologist (SAP or RKK). Pending the report, all patients were given oxytetracycline (Terramycin), a cheap and old broad-spectrum antibiotic. The dose was $500 \mathrm{mg}$ every six hours in LRI and $250 \mathrm{mg}$ every six hours in UTI. If clinical response was satisfactory at the end of 48 hours, the treatment was continued for eight days whatever the sensitivity report. On the tenth day, a senior physician (ASP) evaluated each patient's response as "success" or "failure" depending on the relief of symptoms, regression of neutrophil count, resolution of chest $x$-ray changes in LRI cases, and normalcy of urine on microscopy with either sterile urine or a colony count $<10^{6} / 1$ in UTI cases 24 hours after the last dose. The clinical response of each patient was compared with the reported sensitivity of the isolated pathogen to oxytetracycline (30 $\mu \mathrm{g}$ disc).

Of the 48 patients so studied in one year, 26 had LRI and 22 had UTI. The isolates from LRI cases comprised streptococci, staphylococci, Klebsiella, and Pseudomonas; those from UTI cases comprised $E$ coli, Proteus, Klebsiella, Pseudomonas, and staphylococci. Of 17 LRI patients whose organisms were reported to be resistant, 14 had clinical success so that the incidence of false resistance was $82 \%$; however, of the nine LRI cases in which the organisms were reported sensitive, only two had clinical failure so that the incidence of false sensitivity was $22^{\circ}$. The overall clinical success in LRI was $81^{\circ} \%(21 / 26)$. Of 20 UTI patients with organisms reported as resistant 17 had clinical success so that the incidence of false resistance was $85^{\circ}$; however, of the two UTI patients whose organisms were reported sensitive, both had clinical success. The overall success rate in UTI was $86^{\circ} \%(19 / 22)$.

One possible reason for the high percentage of false-resistant reports (low predictive value) may be that the real frequency of clinically resistant pathogens is much less than the Bauer-Kirby method would indicate." It should be worthwhile to do similar studies with other antibiotics and with other methods such as the Stokes method. ${ }^{3}$ The results, considered along with the pharmacokinetic attributes of the antibiotics, might help to evolve better methods to assess, report, and interpret bacterial resistance in a clinically relevant manner.

A S Phadke $\mathrm{N}$ B JAIN

$S$ A PHADKe

A P SARAF

R K KALBAG G V JOGALEKAR

Departments of Medicine, Microbiology, and Pharmacology,

B f Medical College and Sassoon General Hospitals, Punc 411001 , India

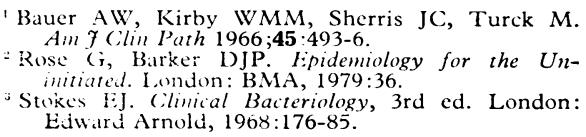

Edward Arnold, 1968:176-85.

\section{Insect-sting encephalopathy}

SIR,-The recent account and subsequent correspondence of central nervous system lesions following insect stings is of interest (2 January, p 20). In the first of the two cases originally reported the offending insect was not named. In the second it was a wasp of unidentified genus and species. No skin test of immediate or other type hypersensitivity reaction was reported, and no class or antigenspecific antibodies to bee or wasp species venom were measured in the sera of these patients. Subsequent correspondence drew attention to the severe neurological sequelae which can follow insect stings (13 February, p 504).

The mechanism of nerve tissue damage in these cases is obscure, with hypoxia appearing to be favoured in the original reports. In the two original cases onset of the condition followed what appeared to be an anaphylactic reaction, in which case hypoxia is the cause to which tissue damage is assigned and in fatal cases the mode of death. Why, in this particular circumstance of anaphylaxis, hypoxia-resistant structures should be selectively damaged remains obscure. Other correspondents have drawn attention to bee stings being associated with the onset of demyelinating illness (13 February, p 504), and in one case a C6 myelitis developed following a bee sting with no associated hypoxic episode.

Bee and wasp venom contain a number of highly basic membrane active peptides, among them mellitin, mast cell degranulating peptide, phospholipase A, and a powerful neurotoxic substance, apamin.' Apamin is a highly basic peptide of 18 amino-acids with two disulphide bridges. It should be stressed that apamin has been isolated only from bee venom to date. Apamin causes clonic convulsions in laboratory animals ${ }^{2}$ and also causes neuromuscular blockade by blocking membrane potassium transfer at neuromuscular junctions. ${ }^{3}$ Bee sting allergy is usually associated with an allergic response to phospholipase A contained in the bee venom. Type 1 or any other type of allergic or alternatively an idiosyncratic reaction to apamin or other membrane active peptides in bee venom has not, to my knowledge, been described. 